\section{Die extrakorporale Kreislaufzirkulation bei der Behandlung von Lungenkrankheiten}

Zusammenfassung: Der Einsatz der extrakorporalen Zirkulation gehört zu den Ausnahmen in der Lungenchirurgie. In dem folgenden Beitrag diskutieren wir anhand einiger klinischer Fälle die Indikationen zum Einsatz der extrakorporalen Zirkulation bei der Behandlung von Lungenkrankheiten. An erster Stelle sind hierbei die pulmonale Embolektomie und die Lungentransplantation zu nennen. Außerdem wird die extrakorporale Zirkulation zur Unterstützung der Oxygenierung während der whole lung lavage bei primärer Alveolarproteinose eingesetzt. Andere, seltenere Indikationen und spezielle chirurgische Eingriffe bei Lungentumoren werden ebenfalls aufgezeigt.

The Extracorporeal Circulation in the Treatment of Pulmonary Diseases: The extracorporeal circulation is seldom used in pulmonary surgery. In this paper, we present some clinical cases and discuss the different indications for extracorporeal circulation in pulmonary diseases. Pulmonary embolectomy and lung transplantation are the main indications for the use of heart-lung-machine. Less frequent indications are oxygen support during whole lung lavage in pulmonary alveolar proteinosis. Lung cancer surgery and other indications for extracorporeal circulation are also discussed.

\section{Einleitung}

Der Einsatz der extrakorporalen Zirkulation (EKZ) ist Routine in der Herzchirurgie, bildet aber in der Lungenchirurgie eine Ausnahme. In dem folgenden Beitrag diskutieren wir die Indikationen der EKZ bei der Behandlung von Lungenkrankheiten anhand einiger Fallbeispiele aus unserer Klinik.

\section{Fall 1}

Ein 30-jähriger Patient erlitt eine Oberschenkelfraktur. Nach der operativen Versorgung der Fraktur und einem Kompartmentsyndrom kam es trotz adäquater Heparinisierung und Frühmobilisation zu einer fulminanten Lungenembolie. Der Patient wurde im Schockzustand in der Klinik aufgefunden und sofort reanimiert. Angesichts des jungen Alters und der instabilen kardiopulmonalen Situation wurde unter fortlaufender Reanimation zur mechanischen Kreislaufunterstüt-

Pneumologie 2001; 55: 409-413

(C) Georg Thieme Verlag Stuttgart · New York ISSN 0934-8387
R. Tosson ${ }^{1}$, K. Klak ${ }^{1}$, E. Sivitanidis ${ }^{1}$, A. Laczkovics ${ }^{1}$,

R. Merget $^{2}$, A. Wiebalck ${ }^{3}$

${ }^{1}$ Klinik für Herz- und Thoraxchirurgie

(Direktor: Prof. Dr. A. Laczkovics),

2 Berufsgenossenschaftliches Forschungsinstitut für Arbeitsmedizin (BGFA), (Direktor: Privatdozent Dr. T. Brüning)

${ }^{3}$ Klinik für Anaesthesie, Intensiv- und Schmerztherapie

(Direktor: Prof. Dr. M. Zenz), Bergmannsheil, Ruhr-Universität, Bochum

zung eine veno-arterielle extrakorporale Membranoxygenierung (ECMO) angelegt [1].

Sofort nach der Implantation der ECMO stabilisierte sich der Kreislauf (43 Minuten nach Beginn der Reanimation). Daraufhin konnte eine Pulmonalisangiographie durchgeführt werden, wobei sich ein kompletter Verschluss der linken Pulmonalarterie darstellte. Aufgrund der Lokalisation der Embolie und des instabilen Kreislaufs wurde unter Einsatz der EKZ eine Embolektomie der Pulmonalarterie durchgeführt [2]. Der Patient konnte am nächsten Tag bei normwertigen Blutgasen extubiert werden und wurde sechs Wochen später bei stabilem Kreislauf (gute arterielle Blutgase und keine Zeichen von Herzinsuffizienz) aus der stationären Behandlung entlassen.

\section{Fall 2}

Hierbei handelte sich um eine 52-jährige Patientin mit pulmonalarterieller Hypertonie und offenem Ductus Botalli (3 cm Durchmesser). Der pulmonalarterielle Druck betrug $165 / 89 \mathrm{~mm} \mathrm{Hg}$ (Mitteldruck $120 \mathrm{~mm} \mathrm{Hg}$ ), der pulmonalarterielle Gefäßwiderstand 1230 dyn.sec. $\mathrm{cm}^{-5}$ und der kapillare Verschlussdruck $30 \mathrm{~mm} \mathrm{Hg}$. Die schwere kardiale Insuffizienz (NYHA III-IV) bei bestehendem Eisenmengersyndrom war nach Ausschöpfung aller konservativen Maßnahmen ausschlaggebend für die Indikation zur Herz-Lungen-Transplantation. Die Operation (Implantation des Herzens und der Lungen) wird unter Zuhilfenahme der EKZ durchgeführt. Fünf Jahre postoperativ zeigt die Patientin weiterhin gute Transplantationsergebnisse.

\section{Fall 3}

Hier beschreiben wir den seltenen Fall einer Patientin mit einer schwergradigen primären Alveolarproteinose (PAP) $[3,4]$. Die 34-jährige Frau war in unser Krankenhaus zwecks Abklärung einer seit 2 Monaten zunehmenden Dyspnoe verlegt worden. Die Röntgenaufnahme des Thorax zeigte diffuse fleckige Verschattungen in beiden Lungen. Die bronchoskopische Diagnostik zeigte die typisch ausgeprägte schaumig-eiweißreiche bronchoalveoläre Lavage (BAL) (Abb.1). In der Lungenbiopsie war die Anreicherung eines feinblasigen, proteinreichen Sekretes mit einzelnen Schaumzellen zu erkennen. Die Patientin entwickelte nach der ersten einseitigen Bronchiallavage eine beatmungspflichtige respiratorische Insuffizienz $\left(\mathrm{PaO}_{2} 31 \mathrm{~mm} \mathrm{Hg}, \mathrm{PaCO}_{2} 28 \mathrm{~mm} \mathrm{Hg}\right)$. Um dennoch eine große therapeutische Lavage durchführen $\mathrm{zu}$ können, wurde eine veno-venöse ECMO angeschlossen. Daraufhin besserte sich sowohl die respiratorische als auch die 


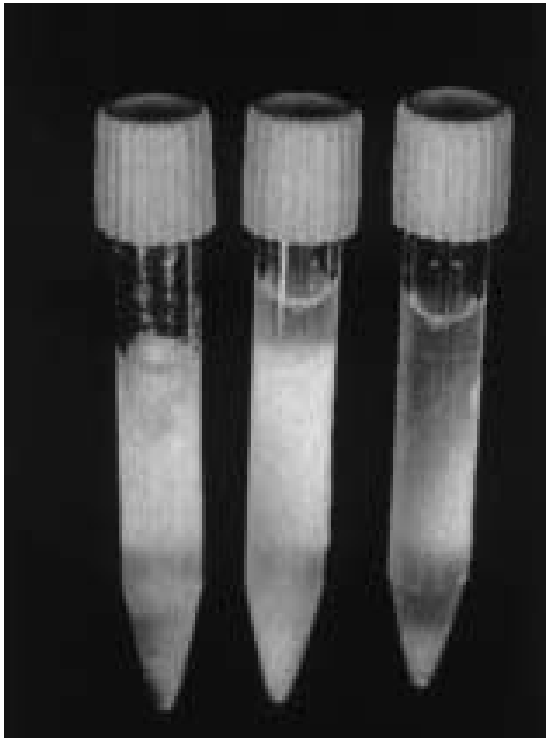

Abb. 1 BAL bei Alveolarproteinose: nach Spülung einer Lunge mit 1 $\mathrm{L}$ (links), mit weiteren $2 \mathrm{~L}$ (Mitte) und nach insgesamt $5 \mathrm{~L}$ (rechts).

kardiovaskuläre Situation der Patientin rasch. Beide Lungen konnten danach ohne Probleme mit mehreren Litern warmer Ringer-Lösung gespült werden (whole lung lavage, WLL). In den nächsten Tagen wurden noch drei weitere WLLs vorgenommen, die dank der ECMO ohne Hypoxämie abliefen. Sieben Wochen nach der ersten WLL konnte die Patientin bei normalen arteriellen Blutgasen $\left(\mathrm{PaO}_{2} 83 \mathrm{~mm} \mathrm{Hg}, \mathrm{PaCO}_{2} 37 \mathrm{~mm} \mathrm{Hg}\right.$ ) ohne Lungenfunktionseinschränkungen entlassen werden.

\section{Fall 4}

Eine 55-jährige Patientin hatte nach einer Hüftoperation chronische Schmerzen und nahm deswegen monatelang nichtsteroidale Antiphlogistika in hoher Dosierung ein. Es entwickelte sich im linken Lungenunterlappen eine Pneumonie, die durch eine gastrobronchiale, transdiaphragmale Fistel infolge eines penetrierenden Ulcus ventriculi hervorgerufen worden war. Den ersten klinischen Hinweis auf diese Fistel erhielten wir nach Platzierung einer Magensonde: Der Beutel füllte und leerte sich atemsynchron. Die Diagnose wurde durch Bronchoskopie und Bronchographie bestätigt. Bei der Einleitung der Narkose kam es zu einem hypoxischen Kreislaufstillstand. Nach der Reanimation fiel trotz Beatmung $\left(\mathrm{FiO}_{2}\right.$ 1,0) der extrem niedrige Sauerstoffgehalt des arteriellen Blutes auf $\left(\mathrm{PaO}_{2} 41 \mathrm{~mm} \mathrm{Hg}, \mathrm{PaCO}_{2} 73 \mathrm{~mm} \mathrm{Hg}\right.$ bei $\mathrm{FiO}_{2}$ 1,0). Daraufhin wurde der Entschluss gefasst, zuerst die ECMO einzusetzen und erst dann die Thorakotomie zwecks Fistelverschluss durchzuführen. Zunächst wurde die veno-arterielle ECMO implantiert, Intraoperativ war eine Fistel zwischen Zwerchfellkuppel und dem linken Unterlappen zu erkennen. Der linke Unterlappen wies eine ausgeprägte Entzündungsreaktion auf. Die Fistel wurde exzidiert, Magen, Zwerchfell und Lunge übernäht. Am 7. postoperativen Tag ging man bei nun stabilem Kreislauf, jedoch weiterhin zu geringer eigener Oxygenierung und um eine Beinischämie zu vermeiden, zu einer veno-venösen ECMO über. Am 9. postoperativen Tag zeigte die Patientin erneut Fieber mit radiologischen Zeichen einer Pneumonie der rechten Lunge. Im Trachealsekret fand sich ein hochresistenter Pseudomonas aeruginosa. Die Patientin verstarb unter dem Bild eines Multiorganversagens bei Sepsis.

\section{Diskussion}

\section{Die Wirkung des extrakorporalen Kreislaufs auf die Lunge}

Durch den ständigen Kontakt des Blutes mit den Fremdoberflächen der Herz-Lungen-Maschine kommt es zur Aktivierung des Komplementsystems, Thrombozytenfunktionsstörungen und verstärkter Bildung von Mediatoren [5]. Es kommt relativ häufig zu Atelektasen, seltener zu akuten Bronchospasmen oder Lungenödem.

Im Allgemeinen ist die ECMO nebenwirkungsarm und gut verträglich. In einigen Fällen kommt es zu therapiebedürftigen Thrombozytenfunktionsstörungen [6]. Wir haben die ECMO bei Patienten mit akutem Nierenversagen und Hämofiltration und bei Patienten mit Gerinnungsstörungen eingesetzt. Unter engmaschiger Kontrolle der Gerinnungsparameter traten keine weiteren Blutungskomplikationen auf und die Patienten konnten schrittweise von der ECMO entwöhnt werden. Diese Methode wird zunehmend auch über längere Zeiträume (mehrere Wochen) eingesetzt [5,6].

\section{Indikationen für den Einsatz der EKZ bei Lungenerkrankungen}

Im Allgemeinen wird der extrakorporale Kreislauf entweder als Bestandteil einer operativen Maßnahme (so genannte Herz-Lungen-Maschine; Fall 2) oder als eine unterstützende Maßnahme bei Herz- oder Lungenversagen z. B. ECMO (Fall 3) eingesetzt.

\section{EKZ bei Lungenembolien}

Eine der bekanntesten und wahrscheinlich die älteste Indikation zum Einsatz der EKZ in der Lungenchirurgie ist die Lungenembolie. Ein Verschluss von Lungenarterien kann das finale Ereignis bei vielen Krankheitsprozessen sein. Klinisch wird die Lungenembolie als Todesursache nur in etwa 10$20 \%$ der Fälle richtig diagnostiziert [7-9]. Durchschnittlich kann man mit einer Häufigkeit von 2,5 bis $5 \%$ tödlicher Lungenembolien im Sektionsmaterial rechnen [4].

Die Lysetherapie ist das Verfahren der Wahl $[2,9,10]$. Die Embolektomie der A. pulmonalis ist heute noch indiziert bei einer fulminanten Lungenembolie mit Kreislaufkollaps und gleichzeitigem Vorliegen einer Kontraindikation zur Lysetherapie [2]. In einer Sammelstatistik wurde eine Überlebensrate nach der chirurgischen Intervention von 55\% errechnet [8].

Lungenembolien können rezidivieren und Ursache einer chronisch thromboembolisch pulmonalen Hypertonie (CTEPH) sein [11,12]. Mit einer pulmonalen Angiographie bzw. CT [13] kann die Diagnose gestellt werden. Bei fehlender konservativer Therapiemöglichkeit stellt sich auch hier die Indikation zur Pulmonalisthrombendarteriektomie [14]. Nach Iversen [15] ist die Indikation zur chirurgischen Therapie gegeben, wenn der Lungengefäßwiderstand in Ruhe auf Werte über 300 dyn.sec.cm ${ }^{-5}$ erhöht ist. Der pulmonalarterielle Mitteldruck liegt dann meistens über $30 \mathrm{~mm} \mathrm{Hg}$ und die Patienten sind herzinsuffizient (meistens NYHA-Stadium III oder IV). 
Bei Patienten mit CTEPH entwickelt sich eine Hyperplasie der bronchialen Arterien, welche die Operation erschweren; indem es beim Eröffnen der pulmonalen Strombahn zu erheblichen retrograden Blutungen aus den bronchialen Arterien kommt. Diese Probleme können bei Kreislaufstillstand in tiefer Hypothermie vermieden werden [15].

\section{EKZ bei Lungentransplantationen}

Die Herz-Lungen-Transplantation (HLTX) und die doppelte Lungentransplantation (beide Lungen als ein Paket durch die transverse Thorakotomie oder mediane Sternotomie) stellen eine klassische Indikation zum Einsatz der EKZ dar [16,17]. Die Ein-Lungen- und die bilaterale (zwei einzelne Lungen bei einem Patienten durch zwei laterale Thorakotomieschnitte) Lungentransplantation stellen eine relative Indikation zum Einsatz der EKZ dar [16].

Bei Ein-Lungen-Transplantation wird der Patient in Halbseitenlage unter Einbeziehung der ipsilateralen Leistenregion gelagert, so dass eine anterolaterale Thorakotomie durchgeführt werden kann und gleichzeitig eine inguinale Kanülierung möglich ist. Zur Überprüfung, ob eine EKZ erforderlich ist, wird die Pulmonalarterie vorübergehend abgeklemmt. Blutdruck, pulmonalarterieller Druck, arterielle und venöse Sauerstoffsättigung werden beobachtet. Bei sinkendem systemischen Blutdruck und $\mathrm{PaO}_{2}$, steigendem pulmonalarteriellem Druck und $\mathrm{PaCO}_{2}$ wird der Anschluss an die EKZ unter Verwendung eines femoro-femoralen Systems vorgenommen.

Die Häufigkeit der Lungentransplantationen wird von der „International Society for Heart and Lung Transplantation“ für 1993 mit ungefähr 1000 angegeben und erreicht damit ihren Gipfel. In späteren Jahren ist die Tendenz rückläufig. Bis Ende 1999 wurden weltweit 2698 Herz-Lungen-, 6514 Ein-Lungeund 4634 bilaterale bzw. Doppellungen-Transplantationen registriert [18].

\section{EKZ bei ARDS}

Beim Lungenversagen ist die ECMO eine Methode zur Unterstützung der Oxygenierung und $\mathrm{CO}_{2}$-Elimination. Die Hauptindikation zum Einsatz der ECMO ergibt sich bei Patienten mit akuter respiratorischer Insuffizienz (ARDS: Adult Respiratory Distress Syndrome), insbesondere, wenn alle konservativen Behandlungsmöglichkeiten ausgeschöpft sind.

Der Anschluss an die ECMO erfolgt meist über eine venovenöse Kanülierung. In der Regel werden hierbei die V. jugularis interna und die V. femoralis als Zugang gewählt. Allerdings ist auch der veno-arterielle oder der arterio-venöse Anschluss möglich über die A. femoralis communis. Laut den Empfehlung der Extracorporeal Life Support Organization (ELSO) ist ECMO indiziert bei akutem, potenziell reversiblem, lebenbedrohlichem Lungenversagen, welches durch konventionelle Therapiemaßnahmen nicht beherrschbar ist $[1,6]$. Wir sehen eine Indikation für die ECMO, wenn $\mathrm{PaO}_{2}<80 \mathrm{~mm} \mathrm{Hg}$ bei $\mathrm{FiO}_{2}>0,6$ oder bei $\mathrm{PaO}_{2}<50 \mathrm{~mm} \mathrm{Hg}$ trotz optimaler Beatmung mit hohem PEEP und bei einer Beatmungsdauer $<1$ Woche. Wir halten bei älteren Patienten (etwa > $65 \mathrm{~J}$ ), Patienten mit schwerer COPD, Patienten mit Gehirnschaden oder Multiorganversagen die ECMO für kontraindiziert. Bei ausreichendem Herzzeitvolumen wird die arteriovenöse ECMO (ECLA: Extracorporeal Lung Assist) bevorzugt. Wird hierdurch die Oxygenierung nicht stabilisiert, kommt die venovenöse ECMO als Alternative in Betracht.

Bei instabilen Kreislaufverhältnissen ist das Standardverfahren die veno-arterielle ECMO. Diese Methode hat jedoch den Nachteil, dass die Lunge nur über die Bronchialarterien mit oxygeniertem Blut versorgt wird. Eine Zusammenfassung der unterschiedlichen Anwendungsverfahren der ECMO und die Indikationen und Kontraindikationen werden in Tab. 1 und $\mathbf{2}$ erläutert. Die Überlebensrate bei Anwendung der ECMO beträgt bei Frühgeborenen $80-94 \%$, im Erwachsenenalter nur noch $28-56 \%[1,6]$.

Die Therapie der ARDS entwickelt sich in zwei Richtungen: einerseits wird durch die heparinbeschichteten EKZ-Systeme und den Einsatz von arteriovenöser ECMO (ECLA ) (s. Tab.1) der Verzicht auf eine volle Heparinisierung der Patienten und eine Vereinfachung der ECMO-Methode $[19,20]$ möglich. Andererseits zeigen sich gute Ergebnisse der konservativen Therapie, z.B. durch Lagerung von Patienten und den Einsatz von neuen Beatmungsmethoden z. B. high-frequency ventilation (HFV) [21-23]. Verschiedene Medikamente werden in der Therapie der ARDS mit guten Ergebnissen eingesetzt, z. B. N0 [4,22] surfactant [25] und Prostaglandin [26]. Verschiedene Studien zeigen, dass NO in der Therapie der ARDS bei den Frühgeborenen gute Ergebnisse erzielt [27]. Lonnqvist [28] fand, dass N0 die Oxygenierung bei Frühgeborenen mit ARDS verbessert, den Bedarf an ECMO um $50 \%$ senkt und die gesamten Behandlungskosten um ca. $60 \%$ erspart.

\section{Seltene Indikationen}

Weiterhin gibt es seltene Indikationen für die ECMO, z. B. die Lungenspülung bei primärer Alveolarproteinose (PAP) (Fall 3). Die venoarterielle ECMO wurde oft bei Patienten mit PAP eingesetzt, meist nur während der WLL $[3,4]$ jedoch ist zu bemerken, dass es nach der Lavage zu vermehrter Hypoxämie kommt. Aus diesem Grund wird heute die venovenöse ECMO empfohlen.

Andere seltene Indikationen zum Einsatz der ECMO sind die Trachearesektion z.B. wegen eines Tumors oder bei akutem Lungenversagen, bei dem ein notfallmäßiger Lungeneingriff nötig ist. Selten ist auch der Abriss der Pulmonalarterie oder des linken Vorhofs beim Absetzen der Pulmonalarterie während onkologischer Operationen. Hier ist die notfallmäßige Versorgung des Vorhofs oder der Pulmonalarterie unter $\mathrm{Zu}$ hilfenahme der EKZ notwendig. Eine umstrittene Indikation zum Einsatz der EKZ stellt die Therapie fortgeschrittener Bronchialkarzinome mit Infiltration in die Aorta dar (IUCC T4 N0 M0) [29].

Über eine modifizierte EKZ kann eine isolierte Perfusion von befallenen Lungensegmenten mit Zytostatika und anderen Medikamenten, ggf. in Verbindung mit Hyperthermie zur lokalen Behandlung von malignen Lungentumoren, vorgenommen werden. Ziel ist die Reduzierung der systemischen Wirkung der eingesetzten Zytostatika, da diese lediglich über die selektiv kanülierten Lungenvenen und -arterien appliziert und nach einer definierten Einwirkzeit mittels Spüllösung wieder entfernt werden können [30-34]. 
Tab. 1 Arten der EMCO

\begin{tabular}{|c|c|c|c|c|c|}
\hline Art der ECMO & Anschluss & Vorteile & Nachteile & Indikation & Material \\
\hline $\begin{array}{l}\text { arteriovenöse ECMO } \\
(\mathrm{ECLA})\end{array}$ & $\begin{array}{l}\text { A. femoralis } \\
\downarrow \\
\text { Oxygenator } \\
\downarrow \\
\text { V. cava inferior }\end{array}$ & $\begin{array}{l}\text { guter } \mathrm{CO}_{2} \text {-Austausch } \\
\text { einfache Implantation }\end{array}$ & $\begin{array}{l}\text { eingeschränkte Oxy- } \\
\text { genierung, } \\
\text { HZV-abhängig, } \\
\text { Ischämiegefahr des } \\
\text { Beines bei Obstruktion } \\
\text { der A. femoralis }\end{array}$ & $\begin{array}{l}\text { Stabile Kreislaufsituation, } \\
\text { gutes Herzzeitvolumen }\end{array}$ & $\begin{array}{l}17 \text { F. arterielle Kanüle } \\
21 \text { F. venöse Kanüle } \\
\text { Oxygenator }\end{array}$ \\
\hline venovenöse ECMO & $\begin{array}{l}\text { V. cava inferior } \\
\downarrow \\
\text { Oxygenator } \\
\downarrow \\
\text { V. cava superior }\end{array}$ & $\begin{array}{l}\text { sehr effizienter } \mathrm{CO}_{2-}^{-} \\
\text {Austausch } \\
\text { hoher } \mathrm{O}_{2} \text {-Gehalt im } \\
\text { pulmonalarteriellen } \\
\text { Blut }\end{array}$ & $\begin{array}{l}\text { mäßige Oxygenierung } \\
\text { des arteriellen Systems } \\
\text { partielle Rezirkulation }\end{array}$ & $\begin{array}{l}\text { bei stabilem Kreislauf und } \\
\text { nicht ausreichender Oxy- } \\
\text { genierung mit arterio- } \\
\text { venöser ECMO }\end{array}$ & $\begin{array}{l}17 \text { bzw } 19 \text { F. venöse } \\
\text { Kanüle (Jugularis inter- } \\
\text { na) } \\
21 \text { F. venöse Kanüle } \\
\text { (V. femoralis) } \\
\text { Oxygenator } \\
\text { Pumpe } \\
\text { Wärmeaustauscher }\end{array}$ \\
\hline venoarterielle ECMO & $\begin{array}{l}\text { V. cava inferior } \\
\downarrow \\
\text { Oxygenator } \\
\downarrow \\
\text { A. femoralis }\end{array}$ & $\begin{array}{l}\text { effizienteste Oxyge- } \\
\text { nierung und } \mathrm{CO}_{2-}^{-} \\
\text {Elimination }\end{array}$ & $\begin{array}{l}\text { inhomogene Verteilung } \\
\text { des oxygenierten } \\
\text { Blutes. } \\
\text { Ischämiegefahr des } \\
\text { Beines bei Obstruktion } \\
\text { der A. femoralis }\end{array}$ & instabiler Kreislauf & $\begin{array}{l}17 \text { F. arterielle Kanüle } \\
21 \text { F. venöse Kanüle } \\
\text { Oxygenator } \\
\text { Pumpe } \\
\text { Wärmeaustauscher }\end{array}$ \\
\hline
\end{tabular}

ECMO: Extrakorporale Membranoxygenierung, ECLA: Extra-Corporeal-Lung-Assist.

Die 17-F-Kanülen implantieren wir mittels Seldinger-Technik. Bei Verwendung von 21-F-Kanülen implantieren wir diese mittels chirurgischer Freilegung der Gefäße.

Tab. 2 Indikationen und Kontraindikationen für die ECMO

ECMO

Indikationen

Akutes, potenziell reversibles, lebensbedrohliches Lungenversagen, welches durch konventionelle Therapiemaßnahmen nicht beherrschbar ist.

Das akute Lungenversagen kann ein

- hypoxämisches Lungenversagen oder

- hyperkapnisches Lungenversagen sein

Kontraindikationen

- Begleiterkrankungen mit zu erwartender kurzer Überlebenszeit

- Begleitende schwere Lungenvorerkrankung

- Multiorganversagen

- ZNS-Beteiligung

- Immunsuppression

- Alter > 65 Jahre

- Kontraindikation zur systemischen Antikoagulation

- Bei v-v-ECMO:

mechanische Ventilation > 7 Tage

gestörte Myokardfunktion

pulmonale Hypertension

Diese Richtlinien sind durch ergebnisorientierte Erfahrungen vorgegeben $[1,6]$. Die Indikation für den Einsatz der ECMO ist individuell zu stellen.

\section{Zusammenfassung}

In der Lungenchirurgie kommt die EKZ nur in ausgewählten Fällen zum Einsatz. Sie ist dennoch von größter Bedeutung, da sie die therapeutischen Möglichkeiten in der Lungenchirurgie maßgeblich erweitert und einigen Patienten erst ein weiteres Überleben ermöglicht.

\section{Literatur}

${ }^{1}$ Alpard S, Zwischenberger J. Adult extracorporeal membrane oxygenation for severe respiratory failure. Perfusion 1998; 13: $3-15$

${ }^{2}$ Peterson K. Acute pulmonary thromboembolism. Circulation 1999; 99: 1280-1287

${ }^{3}$ Rosen S, Castelman B, Liebow A. Pulmonary alveolar proteinosis. N Eng J Med 1958; 258: 1123 - 1142

${ }^{4}$ Sivitanidis E, Tosson R, Wiebalck A, Laczkovics A. Combination of extracorporal membrane oxygenation (ECMO) and pulmonary lavage in a patient with pulmonary alveolar proteinosis. Eur J Cardio-Thorac Surg 1999; 15: 370- 372

${ }^{5}$ Kirklin J, Barratt-Boyes B. Cardiac Surgery. $2^{\text {nd }}$ Ed. USA: Churchill Livingstone, 1998: 61 - 128

${ }^{6}$ Zwischenberger J, Bartlett R. ECMO Extracorporeal Cardiopulmonary Support. In: Critical Care. Ann Arbor: Extracorporeal Life Support Organisation, 1995: 11: 53-86

${ }^{7}$ Lignitz E, Lignitz G, Püschel K. Todesursache Lungenembolie in der Rechtsmedizin. Versicherungsmedizin 1995; 47: 203 - 207

${ }^{8}$ Heinrich F, Klink K. Lungenembolie. Berlin: Springerverlag, 1984: $81-104$

${ }^{9}$ Kienast J, Silling-Engelhardt G. Thrombolysetherapie der Lungenembolie. Internist 1992; 33: 216-221

${ }^{10}$ Meissner E, Niedermeyer J, Worth H, Dorow P, Thalhofer S, Petermann W, Breuer H, Borst M, Oleschewski H, Rasche K, Oertel R, Sill V. Diagnostik der Akuten Lungenembolie. Empfehlung der Deutsche Gesellschaft für Pneumologie. Pneumolog 2000; 12: $587-591$

${ }^{11}$ Reidel M, Stanek V, Widimsky J, Preroesky I. Long term followup of patients with pulmonary thromboembolism. Late prognosis and evaluation of hemodynamic and respiratory data. Chest 1982; 81: 151 - 157

12 Bailey C, Channick R, Rubin L. A new era in the treatment of primary pulmonary hypertension. Heart 2001; 85: $251-252$ 
${ }^{13}$ Dixon J, King M. Chronic thromboembolic pulmonary hypertension. N Eng J Med 2001; 344: 644

${ }^{14}$ Harz RS. Surgery for Chronic thrombembolic pulmonary hypertension. World J Surg 1999; 23: 1137-1147

${ }^{15}$ Iversen S, Hake U, Oelert H. Die chronische Lungenembolie. Dt Ärztebl 1991; 49: 2836-2843

16 Paterson G. Lungtransplantation. In: Pearson F, Deslauriers J, Hiebert C, Ginsberg R, McKneally M (Eds). Thoracic Surgery.. Churchill Livingstone, 1995: 931 -978

17 Patterson G, Cooper J, Dark J, Jones M. Experimental and clinical douple lung transplantiaton. J Thorac Cardiovasc Surg 1988; 95 : $70-74$

${ }^{18}$ Hosenpud J, Bennet L, Keck P, Boucek M, Nowiak R. The Registry of the International Society for Heart and Lung Transplantation: Seventeenth Official Report 2000. J Heart Lung Transplant 2000; 19: $909-931$

${ }^{19}$ Philipp A, Reng M, Behr B, Kaiser M, Birnbaum D. Extrakorporale Lungenunterstüzung ohne Pumpe. Kardiotechnik 1997; 6: 3-5

${ }^{20}$ Bennett C, Johnson A, Field D, Elbourne D. UK Collaborative ECMO Trial Group. Collaborotive randomised trial of neonatal extracorporeal membrane oxygenation: follow-up to age 4 years. Lancet 2001; 357: 1094-1096

${ }^{21}$ Larsson A, Blomqvist H, Frostell C, Linden V, Sjostrand, Walthe. Ventilation in ARDS: respirator, prone position, NO or artifical lung. Lakartidningen 2000; 17: 2058-2063

22 Stelzer H, Kraft P. Improved outcome of ARDS patients: are we really performing better? Intensive Care Med 1999; 25: $887-$ 889

${ }^{23}$ Amato M, Barbas C, Medeiros D, Magaldi R, Schettino G, Filho G, Kairalla R, Deheinzelin D, Munoz C, Oliveira R, Takagaki T, Carvahlo $C$. Effect of a protective-ventilation stragery on the mortality in the ARDS. NEJM 1998; 338: 355-361

${ }^{24}$ Lundin S, Mang H, Smithies M, Stenqvist O, Frostell C. Inhalation of nitric oxide in acute lung injury: results of an european multicenter study. Intensive Care Med 1999; 25: 911 -919

${ }^{25}$ Moller J, Kohl M, Reiss I, Diederich W, Nitsche E, Gopel W, Gortner L. Saline lavage with substitution of bovine surfactant in neonates with mechonium aspiration syndrome (MAS) transferred for ECMO. A pilot study. Crit Care 1999; 3: 19-22

${ }^{26}$ Walmrath D, Schneider T, Pilch J, Grimminger F, Seeger W. Aerosolised prostacyclin in ARDS. Lancet 1993; 342: 961 - 962

${ }^{27}$ Oliveira C, Troster E, Pereira C. Inhaled nitric oxide in the management of persistent pulmonary hypertension of the newborn: a meta-analysis. Rev Hosp Clin Fac Med Sao Paulo 2000; 55: $145-154$

${ }^{28}$ Lonnqvist PA. Efficiency and economy of inhaled nitric oxide in neonates accepted for extra-corporeal membrane oxygenation. Acta Physiol Scand 1999; 167: 175 - 179

${ }^{29}$ Sai N, Maeda M, Miyahara K, Sakurai H, Nakayama M, Takemura $\mathrm{H}$. A case of primary squamous cell lung cancer invading the wall of the distal aortic arch and left subclavian artery: successful surgical management using ECC with selective cerebral perfusion. Nippon-Geka-Gakkai-Zasshi 1998; 99: 264-267

${ }^{30}$ Hipp G, Mauz J. Isolierte Lungenperfusion zur Behandlung von malignen Lungentumoren. Kardiotechnik 1997; 2: 40

${ }^{31}$ Hendriks JH, Van Schil P, Boeck DE, Lauewers G, Van Oosterm P, van Marck E, Eyskens E. Isolated lung perfusion with Melphalan and tumor necrosis factor for metastatic pulmonary adenocarcinoma. Ann Thorac Surg 1998; 66: 1719-1725

32 Johnston M, Christensen C, Minchin R, Rickaby D, Schuller H, Boyd $\mathrm{M}$, Dawson C. Isolated total lung perfusion as a means to deliver organspecific chemotherapy: long-term studies in animals. Surgery 1985; 98: 35-44

${ }^{33}$ Linder A, Friedel G, Fritz P, Kivistö K, McClellan M, Toomes H. The ex-vivo isolated, perfused human lung model: description and potential applications. Thorac Cardiovasc Surgeon 1996; 44: $140-146$

${ }^{34}$ Weksler B, Blumberg D, Lennert J, Ng B, Fong Y, Burt M. Isolated single-lung perfusion with TNF- $\alpha$ in a rat sarcoma lung metastases model. Ann Thorac Surg 1994; 58: 328

\section{R. Tosson}

Klinik für Herz- und Thoraxchirurgie Berufsgenossenschaftliche Kliniken Bergmannsheil Bochum Universitätsklinik Bürkle-de-la-Camp Platz 1 44789 Bochum 